\title{
Nanocomposites for new Functionalities in Multiscale Composites.
}

\author{
Ruggero Volponi ${ }^{1 *}$, Felice De Nicola ${ }^{1}$, Paola Spena ${ }^{1}$ \\ ${ }^{1}$ Italian Aerospace Research Centre, Via Maiorise, 81043 Capua (CE), Italy
}

\begin{abstract}
This work describes the developing of multiscale composites with inherent health monitoring functionality. Nanotubes tend to form electrically conductive networks when embedded even at low concentrations in traditional insulating polymers. By dispersing nanotubes it is possible to obtain a class of polymers that show a piezoresistive behavior. By using that kind of piezoresistive polymer as matrix in fiber reinforced composites, it is possible to develop multiscale composites with an inherent health monitoring functionality. Two different approaches have been developed to obtain a multiscale composite able to monitor the tensional states of composites. In a first manner has been used a thermosetting resin filled with a mixture of nanotubes and graphene and a specific method has been developed to impregnate carbon fiber fabrics with that nanocharged resin. A second idea was to insert a thermoplastic nanocharged thin film into a glass fiber reinforced composites.
\end{abstract}

\section{Introduction}

In recent years the use of fiber reinforced resin composites has continuously expanded especially in aerospace.

Inspection and Maintenance are important aspects when considering the availability of aircraft for revenue flights.

Modern airframe design is exploiting new exciting developments in materials and structures to construct ever more efficient air vehicle able to enable 'smart' maintenance .So there are a wide interest in developing new composites materials in which is the integrations new functionalities.

One of these new integrated functionality studied in these work is the electrical piezoresistivity behaviour of polymer charged with carbon nanotube. [1]

The integration of a nanocharged polymer as matrix into a carbon fiber fabric is possible to obtain a composite in which the intrinsic piezo-resistivity can be used as integrated health-monitoring capability.

\section{Thermosetting matrices}

\subsection{Evaluation of piezoresistivity in epoxy resin charged with carbon nanotube}


In order to develop a multiscale composite we have studied the electrical behavior of an epoxy resin charged with carbon nanotube.

An epoxy resin, charged with carbon nanotube, becomes electrical conductive. Furthermore, an epoxy resin charged with carbon nanotube show a heavy piezo-resistivity effect: his electrical resistance change when is applied a strain.

So the idea is to use that nano-composite as matrix in a carbon fiber reinforced composite to add an intrinsic health-monitoring functionality.

The epoxy resin system chosen, is sold by Elantas with the name of EC327

That resin has a very low viscosity at room temperature, around $300 \mathrm{mPas}$, a long pot-life of several hours at room temperature, a storage modulus of $3200 \mathrm{Mpa}$ and a glass transition temperature of about $190^{\circ} \mathrm{C}$.

The resin has been charged with carbon nanotube 3100 Nanocyl through a mechanical system: a three roll mill Exakt 80E, with three different amount of CNT: $0,3 \%, 0,6 \%$, $0,9 \% \cdot[2]$

The cure of nano-charged resins followed the proper cure cycle as given by data sheet.

On different samples have been done the electrical contacts using a conductive silver paste at the edge of each sample. Then they are integrated in the pure resin to insulate and to have a mechanical clamping. (Fig.1)

Electro-mechanical tests have been performed over the sample using an Instron tensile machine and a Keithley 2182A picoammeter with a nanovoltmeter Keithley 6221

An extensometer staked over the sample measured the strains recording simultaneously the variation of electrical resistance (Fig.2).

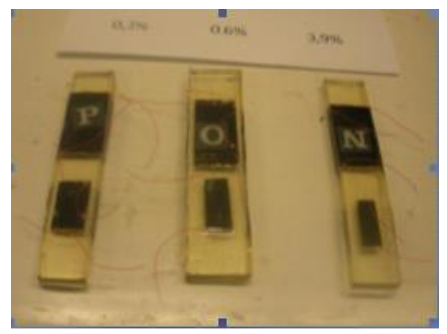

\section{Error! Objects cannot be created from editing field codes.}

Fig.2 Setup for Electro-mechanical tests

Fig.1 Sample of nanocharged resins

Two different kind of test have been performed: first a load-unload cycles and a creep test (load, maintain, unload).

A very important parameter estimated in the tests was the Gauge factor $\mathrm{G}_{f}$ defined as:

$$
G F=\frac{\Delta R / R_{G}}{\epsilon}
$$

It account the variation of the electrical resistance due to the strain. If the Gauge factor is greater than 1 means that there are an amplifier effect.

So for resin charged using $0,3 \%$ of CNT the response were: 


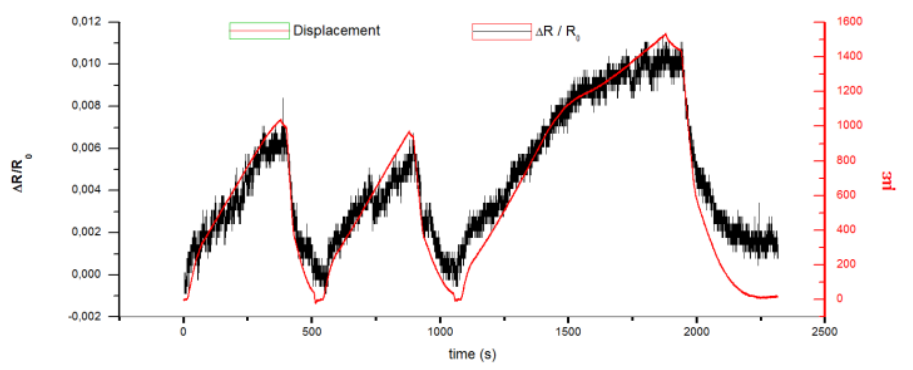

Figure 3 Cycles of load and unload: evaluation of piezoresistivity in resin E327 with 0,3\% CNT

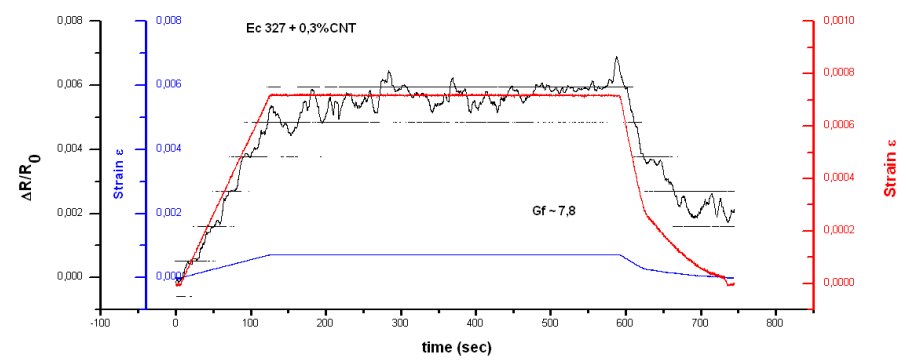

Figure 4 Creep test: evaluation of piezoresistivity in resin E327 with $0,3 \% \mathrm{CNT}$

Similar tests have been performed over samples charged with $0,6 \%$ and $0,9 \%$ of CNT in a range of strains that not exceeds $0,15 \%$.

The following table summarize the Gauge factors, the electrical conductivity and the viscosity measured varying the charge:

Table 1 Gauge factors, Conductivity and Viscosity of nanocharged resins

\begin{tabular}{|c|c|c|c|}
\hline Sample & Gauge Factor & Conductivity (S/m) & $\begin{array}{c}\text { Viscosity@1Hz@ } \\
80^{\circ} \mathrm{C} \\
\left(\mathrm{Pa}^{*} \mathrm{~s}\right) \\
\end{array}$ \\
\hline $\mathrm{EC} 327+0,3 \%$ & 8 & $3 e-4$ & 1,3 \\
\hline EC $327+0,6 \%$ & $\sim 4$ & $1,3 \mathrm{e}-2$ & 2 \\
\hline $\mathrm{EC} 327+0,9 \%$ & 3 & $1,4 \mathrm{e}-1$ & 30 \\
\hline
\end{tabular}

It's possible note that the Gauge factor decrease as the charge increase. This comportment is due to the realization of the conductive network by nanotubes. These conductive network follows a percolative so at low ratio of carbon nanotubes is more sensitive to the variations of shape of the sample due to the mechanical stresses. [3]

\subsection{Manufacturing of a panel}

For the definition of a CFRP manufacturing processes really applicable first of all is fundamental to study the rheological characteristics of the nanocharged resins proposed. 
From this study is possible to evaluate the viscosity and the available time for the fabrication of composite (pot-life).

On those parameters is possible to select the method of manufacturing of composites.

A very common used process is the liquid infusion where an usual values of viscosity accepted is near $300 \mathrm{mPas}$, with an injection temperature not exceeding $80^{\circ} \mathrm{C}$. In literature is possible to find a theoretical maximum limit of $0.8 \mathrm{~Pa}$ s. [4] Often a nanofilled resin exceeds the limit of $0.8 \mathrm{~Pa} \mathrm{~s}$; then the usual liquid infusion becomes unfeasible. To maintain the viscosity value as low as possible, the amount of nanotubes to disperse into the resin has been chosen as $0,3 \%$.

However the value of viscosity remain too high for an infusion process.

To overcome this critical point a variation of Resin Film Infusion (RFI) is been used, spreading a thick film of nanocharged resin over a ply $(400 \mathrm{~mm} \times 400 \mathrm{~mm})$ of carbon fiber cloths and a dry preform made by laminating 12 plies of carbon fiber cloths has been placed on the resin thick film and over than has been positioned a single layer of glass fiber to interrupt the electrical contact between the carbon plies:

Finally, over a further layer of carbon, another thick film of resin was placed and additional 12 carbon plies were added. Then the edges of the preform have been sealed to force the resin to flow through the thickness. The laminate has been covered by a porous release film and a distribution medium to allow the resin to escape from the upper side. A bleeder medium has been placed around the preform to receive the excess of resin, and a vacuum bag has been prepared. The closed laminate has been placed into autoclave.
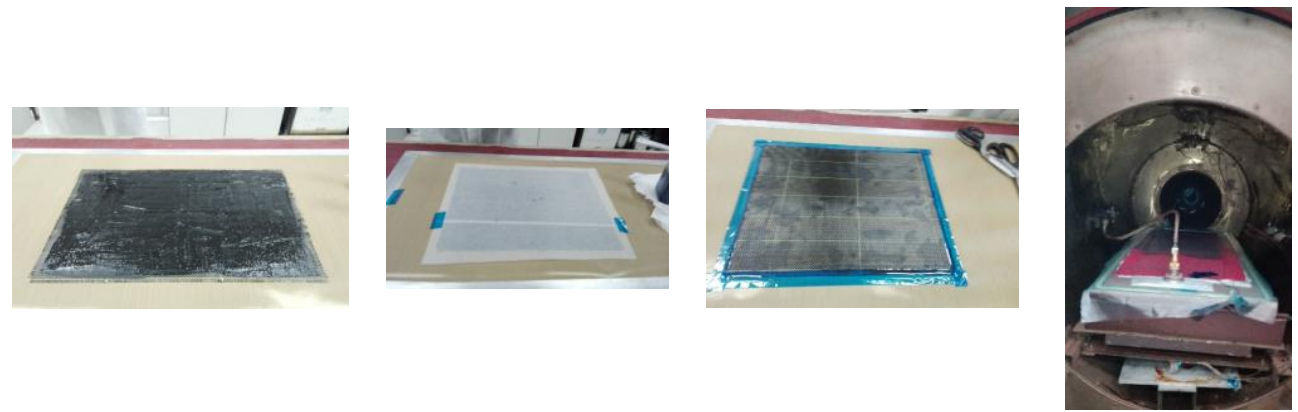

Figure 5 Phases of manufacturing of panel

The quality of manufacturing has been verified cutting an edge of panel and observing it means an optical microscope at different magnitudes:

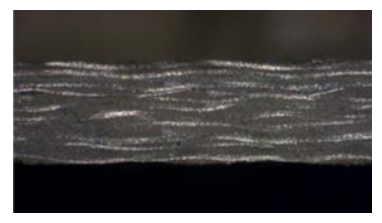

$25 \mathrm{X}$

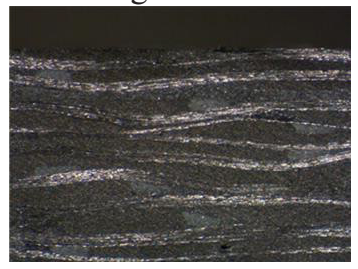

$150 \mathrm{X}$

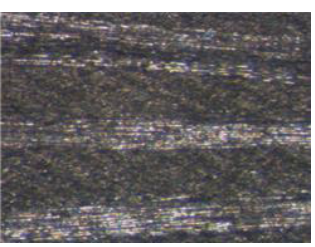

$350 \mathrm{X}$

Figure 6 Inspections with optical microscope at different magnitudes

The microscope analysis shows that the panel does not have macroscopic defects and is uniformly infiltrated; therefore it can be asserted that the manufacturing technique developed for the realization of fiber-reinforced panels with nanocharged matrices has confirmed its effectiveness. 


\subsection{Evaluation of strain sensing capabilities}

Electro-mechanical tests have been performed over the panel using an Instron tensile machine and a Keithley 2182A picoammeter with a nanovoltmeter Keithley 6221. Strains have been measured by a extensometer staked under the sample and the variation of electrical resistance has been recorded simultaneously using a Vishay mod 5100 con High Level Card 5130.

Electrical contacts have been made on two sides and opposite sides of the panel using a conductive paste.

Tests have been performed bending the panel in a three point flexure mode configuration.

Two different tests were performed applying an increasing force up to $1 \mathrm{KN}$, then the load has been kept constant for about 400s in the first test. In a second test has been applied a similar scheme of loading, but the force has been kept constant for $120 \mathrm{~s}$.

Figure 7 Three point bending test

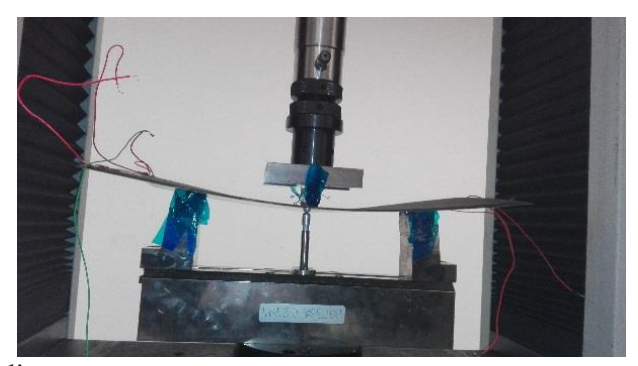

The following pictures shows the results obtained in the two tests:



Figure 8 Response of piezoresistivity $1^{\circ}$ test 


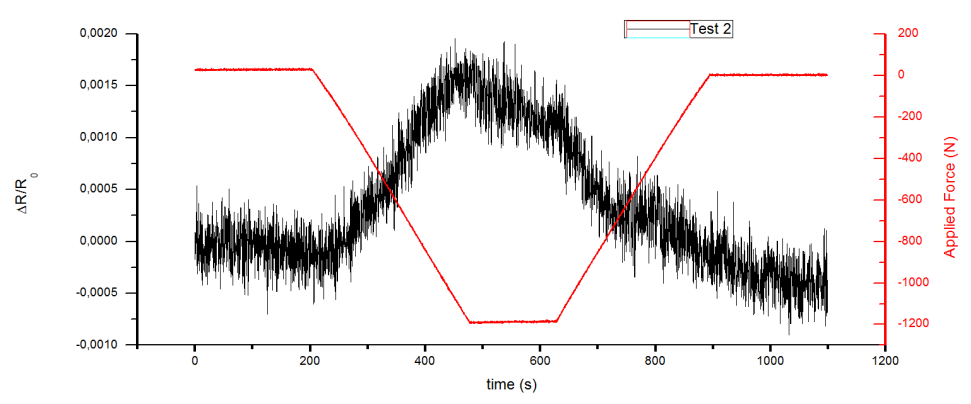

Figure 9 Response of piezoresistivity $2^{\circ}$ test

Data shows that the effect of piezoresistivity is present in both tests but is of weak entity; moreover, in the second test the signal is further attenuated.

It's possible to link that evidence to the fact that the piezoresistive effect is concentrated in the nanocharged resin and the presence of carbon fibres reduces this behaviour, however remains noticeable thanks to the small amount of nanocharged resin impregnated in the layer in glass layer.

\section{Inner thermoplastic layer}

An alternative solution to chain the piezoresistivity properties of a nanocharged polymer into a fiber reinforced composite to get an integrated health-monitoring system is based on a nanocharged thermoplastic polymer sheet that can be easily integrated during the laminating phase in the manufacturing process of epoxy resin fibers reinforced composite . The selection for a suitable material has been done over a wide class of thermoplastic polymers and finally has been chosen a polymer based on a mixture of phenoxy resins which is soluble with epoxy resins. [5]

\subsection{Fabrication of nanocharged thin sheet}

The pristine material, Grilon ${ }^{\circledR}$, produced by EMS-Grivory is a yarn that isn't soluble in any common solvent, so to add nanotubes to it it's necessary to use an extrusion process.

The process of extrusion is done by means an extruder, that heat the polymer over his melting temperature and mix it through two screws that rotates in opposite direction.

The temperature used to mix and to extrude the polymer with nanotubes has been settled at $160^{\circ} \mathrm{C}$, where the viscosity of the nanocharged polymer is appropriate to be extruded. Pellets of polymer charged with $5 \%$ and $10 \%$ with nanotubes were extruded.

From each kind of pellets has been manufactured several thin sheets by means of an hot press.

The thickness of the films are about $150 \mu \mathrm{m}$ for that charged with $5 \%$ and about $300 \mu \mathrm{m}$ for films obtained from pellets charged with $10 \%$ of CNT.

\subsection{Characterization of thin films: Piezoresistivity and adhesion}

The films have been evaluated over different aspects: their electrical properties and their compatibility with epoxy resin. 
The characterization of the electrical proprieties of the sheets has gone on to evaluate the piezoresistivity of the sheets.

The samples have been bonded with a structural adhesive over an aluminium plate.
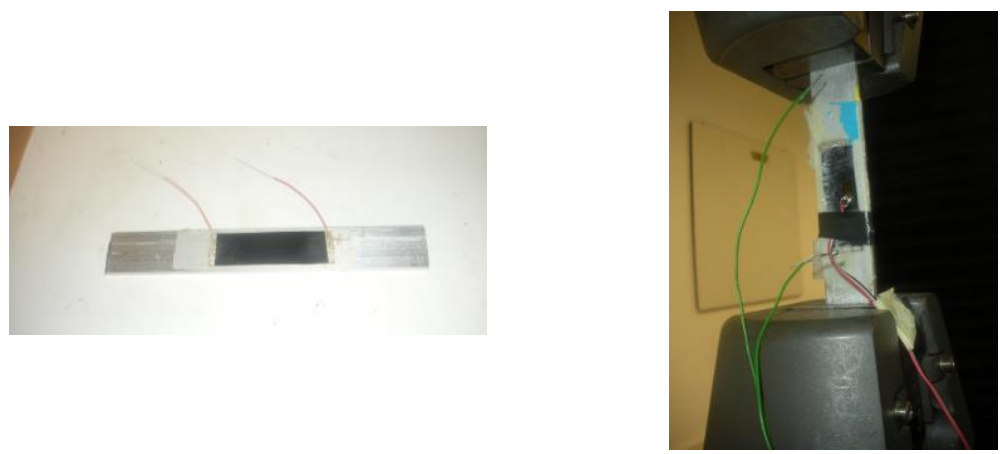

Figure 10 Sample

Strains have been applied to the sample by a mechanical testing machine INSTRON 4505 and in the same time a constant current flows across the sample.

Simultaneously strains and tension have been recorded in the time:

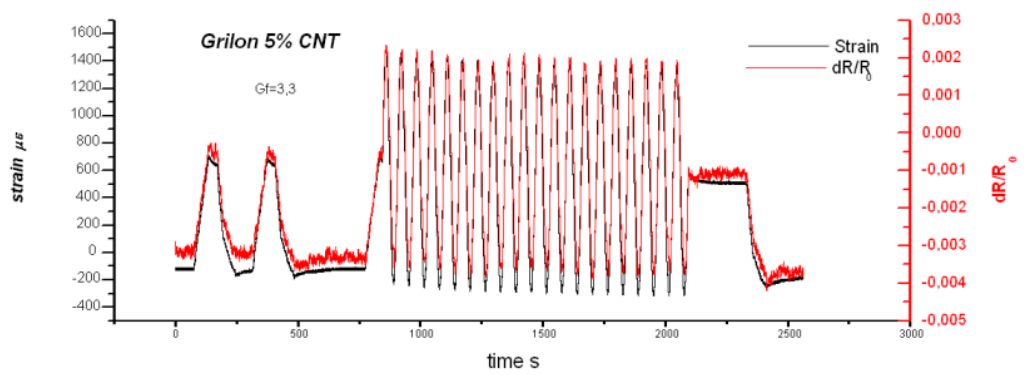

Figure 11 Tests for 5\% CNT charged layer

The Gauge Factor found for the sample with 5\% of nanotubes is around 3.3, appropriate to develop a strain sensing system.

The properties of adhesion of a nanocharged thin sheet to the epoxy resin matrix in a fiber reinforced composite have been evaluated following the standard ASTM D 2344 that describe how to perform a Short Beam Strength test on a laminate.

A layer of pristine Grilon of thickness of $147 \mu \mathrm{m}$ and another one of Grilon charged with $5 \%$ of nanotubes and thick $156 \mu \mathrm{m}$ are put in the middle of a laminate of 20 carbon prepreg plies ( Hexcel M18), in different zones and leaving a zone without any kind of layer for reference. From each of three different areas has been cut 4 sample and for each one have been put over two edges and in the middle has been applied a load (Fig10 A).

From the maximum load applied during the test is possible to evaluate the Strength of short beam FSB: 


$$
F_{S B}=0.75 \frac{P_{M}}{b \cdot h}
$$

where $P_{M}$ is the maximum load allied, $\mathrm{b}$ the width of the sample and $\mathrm{h}$ the thickness

The follow pictures shows how the short beam strength have been performed and the values of the Strength $F_{S B}$ evaluated over four samples tacked from the three different region of the composites.

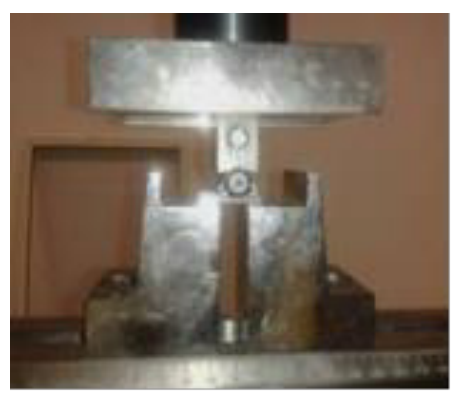

Figure 12 Short Beam Test: Setup

The red values are the strengths for the composite with a pristine layer of Grilon in the middle, the green values are the strengths for the composites with a layer of Grilon charged with $5 \%$ of nanotubes, and the black values are the strengths for the composite without layer.

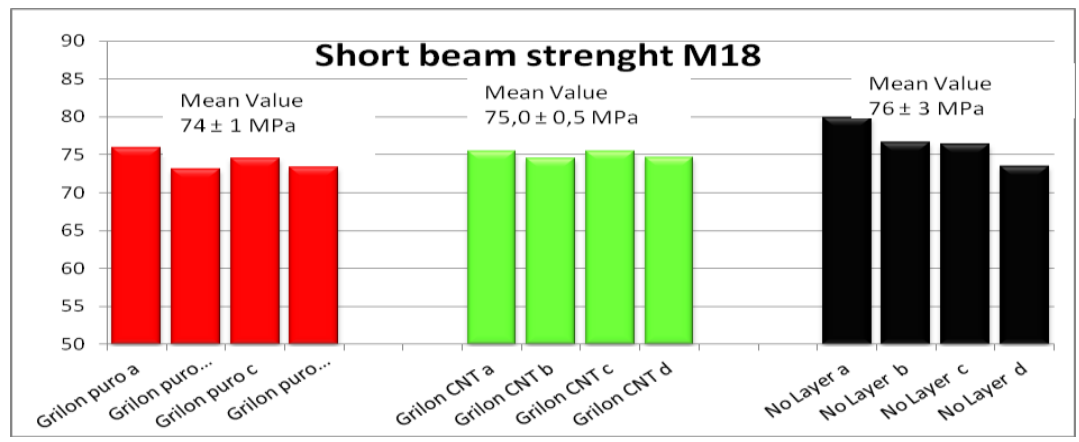

Figure 13 Short Beam Test: Comparison of the Values of the Strengths

That values highlight a good adhesion of the nanocharged layer to the matrix of the laminate and isn't source for delamination

\subsection{Manufacturing and test of a panel with health-monitoring embedded system}

The integration of a nanocharged thin sheet in a composite has been done as the last step to verify the possibility to use it as an integrated health-monitoring system. 
A $20 \times 20 \mathrm{~cm}$ panel has been manufactured by means of hand lay-up process, using glass fiber plies and a commercial epoxy resin.

The nanocharged film with $5 \%$ of nanotubes has been put in the middle of the 24 plies of the panel. The piezo-resistive sheet has three electrical contact in each lateral sides. After the laminating phases the panel has been cured in autoclave under a vacuum bag.
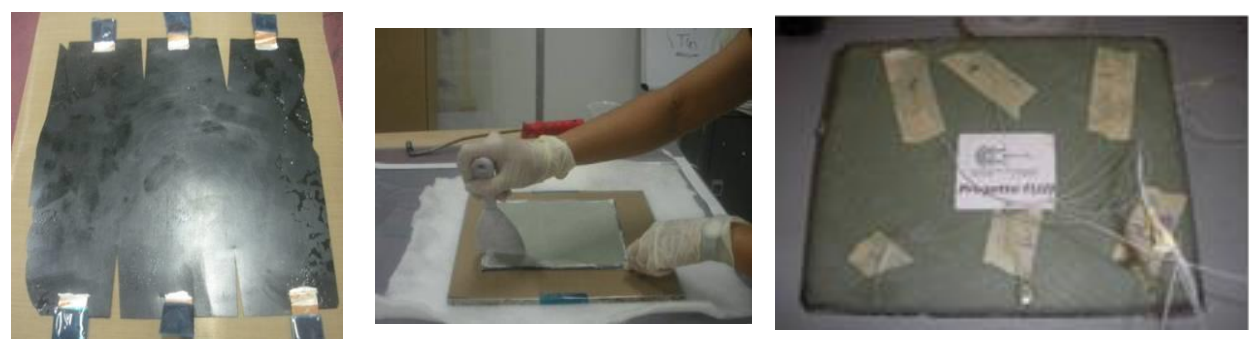

Figure 14 : Phases of manufacturing of glass fiber reinforced panel with nanocharged layer

The panel has been tested with a mechanical testing machine model MTS 810 with a load cell of $250 \mathrm{KN}$. Tests have been done bending the panel in a three point flexure mode During the tests a continuous current of $0,5 \mathrm{~mA}$ flew between three different points of the panel, and simultaneously were recorded the tensions between that points, the displacement of the load cell and the force applied.

The following picture shows how the signals of the variation of resistance follows the displacement and this means that the method could be used as an embedded healthmonitoring system.

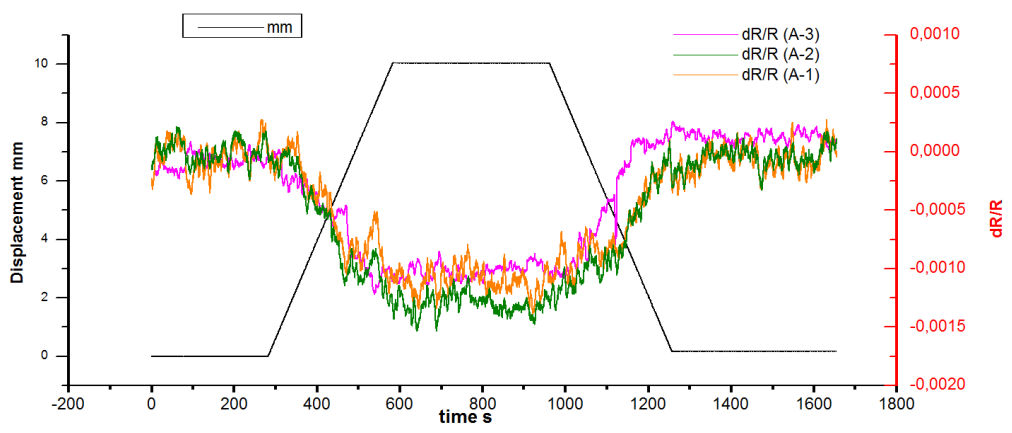

Figure 15 Response of electrical resistance while a load has been applied

\section{Conclusions}

The aim of this work was to show two different approches developed at CIRA to manufacture a multiscale composites with new inherent functions through the integration of nanocharged matrices in fiber-reinforced systems.

The work done to create a fiber-reinforced composite with a thermosetting nanocharged matrix is still applicable to forms having a large usable surface, usually flat, that allows an infusion through its thickness. In this way, is possible to minimize the infusion path, and to solve the filtering problem that the dry preform acts on the nanocharged resin. 
The success of the process is also evidenced by the microscope investigation.

The test of health monitoring capability through the piezoresistive matrix' propriety resulted lessened by the presence of carbon, but this lessening could be reduced adding more insulating layers in the composite.

Differently, the research on the possibility to have a thermoplastic piezoresistive system has given a very interesting result. The film solution made of phenoxy resins, loaded with nanotubes, has demonstrated being an efficient way to integrate nanoparticles into an epoxy matrix composite.

It has been observed that the nature of the phenoxy resins is completely compatible with the epoxy matrix, to the point that a complete blending of phenoxy resin and epoxy matrix is possible. Moreover that solution is totally integrable with different manufacturing process of composites as lamination or infusion, as well as being able to work as integrated system for strain monitoring suffered by the composite itself, thanks to its piezoresistive nature.

\section{References}

[1] Wichmann, M. H. G.; Buschhorn, S. T., Gehrmann, J., \& Schulte, K. (2009). Phys. Rev. B, Vol. 80, (December 2009), 245437, ISSN 1098-0121

[2] Iosif D.; Rosca, Suong V., Hoa. (2009) Carbon, Vol.47, Issue.8, (July2009), pp. 1958-1968, ISSN 0008-6223

[3] R. Volponi - P. Spena - F. De Nicola - A. Grilli ECCM16 - 16TH EUROPEAN CONFERENCE ON COMPOSITE MATERIALS, Seville, Spain, 22-26 June 2014

[4] L. Guadagno, U. Vietri, M. Raimondo L. Vertuccio, G. Barra, B. De Vivo,P. Lamberti, G. Spinelli, V. Tucci, F. De Nicola R. Volponi, S. Russo Composites Part $B$ Engineering 05/2015; 80

[5] L. L. P. T. M. T. P. P. J. H. Doris W.Y. Wong Composites Part A: Applied Science and Manufacturing, pp. Pages 759-767, Volume 41, Issue 6, (June 2010) . 